\title{
Triptolide induces apoptosis in human anaplastic thyroid carcinoma cells by a p53-independent but NF-kB-related mechanism
}

\author{
WENBO ZHU ${ }^{1 *}$, HAIYAN HU ${ }^{2 *}$, PENGXIN QIU ${ }^{1}$ and GUANGMEI YAN ${ }^{1}$ \\ ${ }^{1}$ Department of Pharmacology, Zhong-shan School of Medicine, \\ ${ }^{2}$ School of Pharmaceutical Sciences, Sun Yat-Sen University, Guangzhou 510080, P.R. China
}

Received June 11, 2009; Accepted August 5, 2009

DOI: $10.3892 /$ or_00000580

\begin{abstract}
Triptolide, a traditional anti-inflammatory and antiimmunodepressive agent, has been reported to exert antineoplastic activity on several human tumor cell lines. This study investigates the pro-apoptotic function and the functional mechanism of triptolide on anaplastic thyroid carcinoma (ATC) cells. Experiments presented here demonstrated that triptolide had dose-dependent effects on cell viability of human ATC cell line TA-K cells through inducing cell apoptosis. In the molecular level, triptolide did not successfully initiate p53 signaling pathway, but downregulated the nuclear factor $\kappa \mathrm{B}$ $(\mathrm{NF}-\kappa \mathrm{B})$ pathway. Our studies suggest that triptolide functions as an effective apoptotic inducer in a p53-independent, but $\mathrm{NF}-\kappa \mathrm{B}-$ dependent mechanism, thus providing a promising agent for tumor types with p53 mutation/deletion.
\end{abstract}

\section{Introduction}

Anaplastic thyroid carcinoma (ATC) is one of the most aggressive and lethal human cancers (1). Most patients with ATC have an extremely poor prognosis due to its rapid and pathological growth (2). This malignancy is unsuitable for radical resection and seems completely refractory to the non-surgical therapies such as radiation therapy and chemotherapy $(3,4)$. Efforts to elucidate the molecular mechanisms related to the tumor's aberrant growth and drug resistance are urgently needed in order to develop innovative and effective treatments.

Suppression of apoptosis, together with deregulated cell proliferation, are critical events in tumor progression (5). Commitment of cells to apoptosis is governed largely by

Correspondence to: Dr Guangmei Yan, Department of Pharmacology, Zhong-shan Medical College, Sun Yat-Sen University, 74 Zhongshan Road II, Guangzhou 510080, P.R. China

E-mail:ygm@mail.sysu.edu.cn

${ }^{*}$ Contributed equally

Key words: triptolide, apoptosis, anaplastic thyroid carcinoma, p53, nuclear factor $\kappa \mathrm{B}$ protein-protein interactions between members of the Bcl-2 protein family, which is categorized into two groups: proapoptotic oncoproteins such as Bax, Bad and anti-apoptotic ones such as $\mathrm{Bcl}-2, \mathrm{Bcl}_{\mathrm{L}}$ (6). Bax homodimers promote apoptosis, while $\mathrm{Bax} / \mathrm{Bcl}-2$ heterodimers suppress apoptosis, leading to the idea that the relative level of these two proteins determines the destiny of cells to live or to proceed to apoptosis. In the case of tumors, the anti-apoptotic proteins have been found to be overexpressed, causing aberrant proportion and abnormal cell survival (7). Thus, an obvious strategy for cancer therapy is to induce apoptosis through increasing the relative ratio of pro- and anti-apoptotic proteins in tumor cells.

Resistance to apoptosis is a key factor preventing response to chemotherapy drugs in thyroid cancer and many other cancers. Cancer cells have several survival mechanisms that help them elude cell death induced by chemotherapeutic drugs, among which, mutant p53 is of particular importance (8). In human tumors, the outcome of $\mathrm{p} 53$ reactivation is to induce apoptosis of abnormally proliferating cells, which largely depends on its downstream effector genes such as cyclindependent kinase inhibitor $\mathrm{p} 21$, pro-apoptotic protein Bax and so on (9). To restore p53 expression and its transcriptional function, or to develop a p53-independent and pro-apoptotic target becomes a promising modality for good response to chemotherapy agents.

Nuclear factor $\kappa \mathrm{B}(\mathrm{NF}-\kappa \mathrm{B})$ is active in most tumor types, and regulates a series of pivotal events in the tumor progress including cell survival, cell proliferation, angiogenesis and invasion (10). For cell survival, NF- $\kappa \mathrm{B}$ mainly suppresses cell apoptosis by increasing the transcription of genes encoding anti-apoptotic proteins, for instance Bcl-2 and $\mathrm{Bcl}-\mathrm{X}_{\mathrm{L}}$ (11). Thus, suppression of NF-кB pathway should be effective to induce apoptosis of tumor cells.

Triptolide is a small molecule and a natural product, which is extracted from the Chinese medicinal herb Tripterygium wilfordii Hook F (TWHF). Since the 1960s, triptolide has been used as anti-inflammatory and anti-immunity agents, especially in the treatment of rheumatoid arthritis and systemic lupus erythematosus (12). With increasing research, triptolide has been demonstrated to have anti-neoplastic activity mainly via inducing apoptosis in various cancer types (13-16). We analyzed the pro-apoptotic effect of triptolide on 
human anaplastic thyroid carcinoma TA-K cells and explored the possible mechanism involved.

\section{Materials and methods}

Cell culture and drug treatment. Human ATC cell line TA-K cells were maintained in William's medium $\mathrm{E}$ with $10 \%$ fetal bovine serum (FBS) (Invitrogen, Grand Island, NY). Cells were cultured in a $5 \% \mathrm{CO}_{2}$-humidified atmosphere at $37^{\circ} \mathrm{C}$. Triptolide (Sigma-Aldrich, St. Louis, MO) was dissolved in DMSO to obtain $1 \mathrm{mM}$ stock solution and then was added in medium at indicated concentrations for indicated time.

MTT assay. Cells were incubated with various concentrations of triptolide for indicated time. Then $20 \mu 1$ 3-(4, 5-dimethylthiazol-2-yl)-2, 5-diphenyl tetrazolium bromide (MTT) solution (Sigma, $5 \mathrm{mg} \mathrm{ml}^{-1}$ in PBS) was added to induce the production of formazan crystals. MTT solution was aspired off after $4 \mathrm{~h}$ and $100 \mu 1$ dimethyl sulfoxide (DMSO) was added to solubilize the formazan crystals. The optical density (OD) was determined at $570 \mathrm{~nm}$ using an EXL800 microimmunoanalyser (BioTek, Burlington, VT). The cell viability rate = OD experiment/ODcontrol x $100 \%$.

Hoechst 33258 staining and transmission electron microscopy. Hoechest 33258 staining was performed according to the method suggested by the manufacturer. In brief, cells were fixed with $4 \%$ paraformaldehyde for $10 \mathrm{~min}$ and then stained with Hoechest 33258 (Sigma, $5 \mu \mathrm{g} / \mathrm{ml}$ ) in the dark. The chromosomal DNA morphology was observed by fluorescence microscopy with a $340 \mathrm{~nm}$ excitation filter. Transmission electron microscopy (TEM) was conducted as described (17).

Western blot analysis. Cells treated with triptolide for indicated time were scraped and then resuspended in protein extraction reagent. The cell lysate was centrifuged at 140,000 $\mathrm{g}$ for $10 \mathrm{~min}$ at $4^{\circ} \mathrm{C}$ and the supernatant was collected and stored at $-70^{\circ} \mathrm{C}$. The concentration of protein was determined using BCA protein assay kit according to the manufacturer's instructions. Equal amounts of protein $(60 \mu \mathrm{g})$ was separated by $12 \%$ SDS-PAGE. After electrophoresis, the proteins were transferred to PVDF membrane, blocked with 5\% skimmed milk in TBS for $2 \mathrm{~h}$, reacted with antibodies against p53 (1:1000, Cell Signaling Technology, Beverly, MA), p21 (1:1000, Cell Signaling Technology), Bax (1:1000, Cell Signaling Technology), p65 (1:200, Santa Cruz, CA), Bcl-2 (1:1000, Cell Signaling Technology), Bcl-X $\mathrm{L}_{\mathrm{L}}(1: 1000$, Cell Signaling Technology), B-actin (1:1000, Thermo Scientific IHC, Fremont, CA) or tubulin (1:5000, Sigma) overnight. After reaction with horseradish peroxidase-labeled secondary antibody, the immune complexes were visualized using the ECL-detection reagents following the manufacturer's procedure.

Statistical analysis. Data are presented as mean \pm standard deviation (SD) of three separate experiments. Student's paired t-test was used to compare two sets of data from the same subjects. A result with a P-value of $<0.05$ was considered statistically significant.

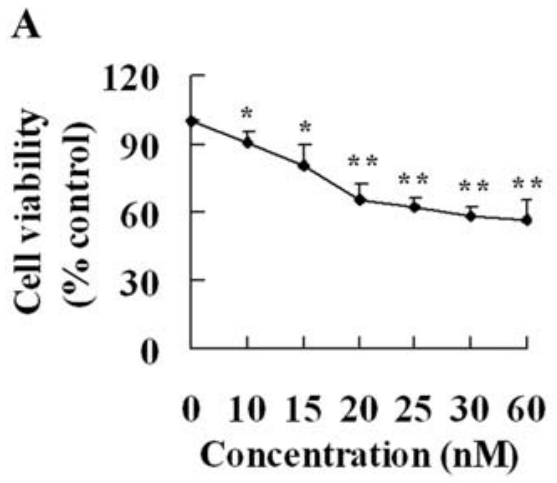

B

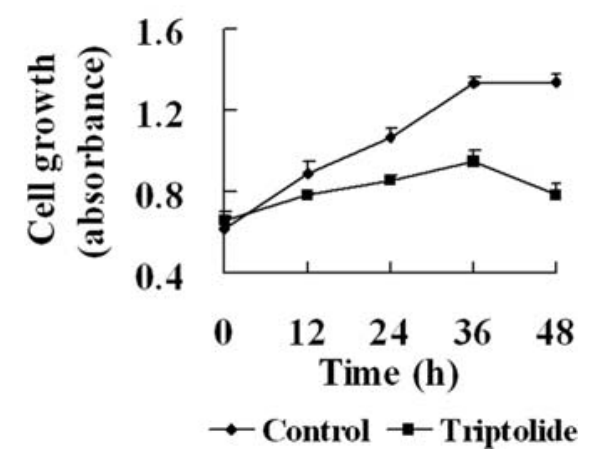

Figure 1. Effects of triptolide on the cell viability and cell growth of TA-K cells. (A) The concentration-dependent effect of triptolide on the cell viability. Cells were treated with various concentrations of triptolide and cell viability was determined using the MTT assay at $48 \mathrm{~h}$. The cell viability rate $=\mathrm{OD}$ experiment/ODcontrol x 100\%. ${ }^{*} \mathrm{P}<0.05,{ }^{* *} \mathrm{P}<0.01$ compared with the control. (B) The effect of triptolide on the cell growth. Cells were treated with $30 \mathrm{nM}$ triptolide for 12,24,36 and $48 \mathrm{~h}$ and cell growth was measured by MTT assay.

\section{Results}

Triptolide reduces cell viability dose-dependently and inhibits cell growth in TA-K cells. Using MTT assay, we determine the cell viability and cell growth of TA-K cells. A dose-related inhibitory effect on cell viability was observed after exposure to triptolide in the 10-60 nM range for $48 \mathrm{~h}$ (Fig. 1A). From $20 \mathrm{nM}$ on, this inhibitory effect of triptolide gradually leveled off. In addition, as shown in Fig. 1B, the cell growth of the $30 \mathrm{nM}$ triptolide-treated group is significantly slower than control in the time range of 12 to $36 \mathrm{~h}$. After $36 \mathrm{~h}$, the amount of cells in the treated group was gradually reduced, indicating the existence of cell death induced by triptolide.

Triptolide induces apoptosis of TA-K cells. Cell death can contribute to either apoptosis or necrosis. To distinguish whether triptolide induces apoptosis or necrosis in TA-K cells, we stained the nuclear of TA-K cells cultured with $30 \mathrm{nM}$ triptolide for $48 \mathrm{~h}$ with Hoechest 33258. Fig. 2A clearly shows that the nucleus of apoptotic cells display characteristics of morphological alterations such as condensed nuclei and cell shrinkage in triptolide-treated group. To further confirm this result, we fixed the untreated and treated cells and observed cell morphology using transmission electron microscopy. Typical apoptotic bodies confirmed apoptosis in cells treated with triptolide for $48 \mathrm{~h}$ (Fig. 2B). 
A
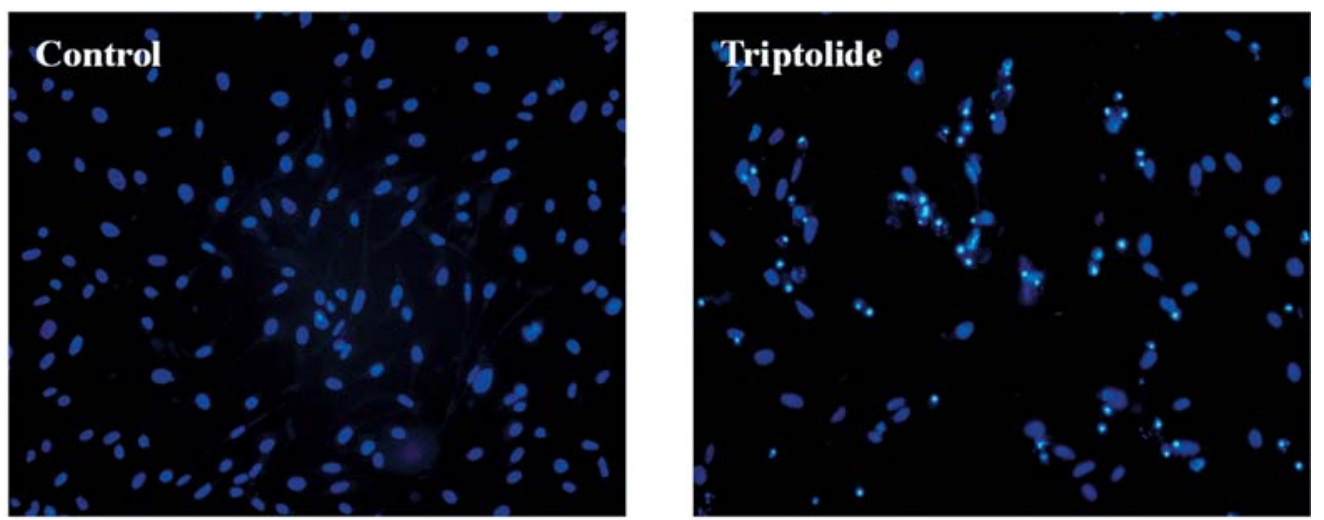

B
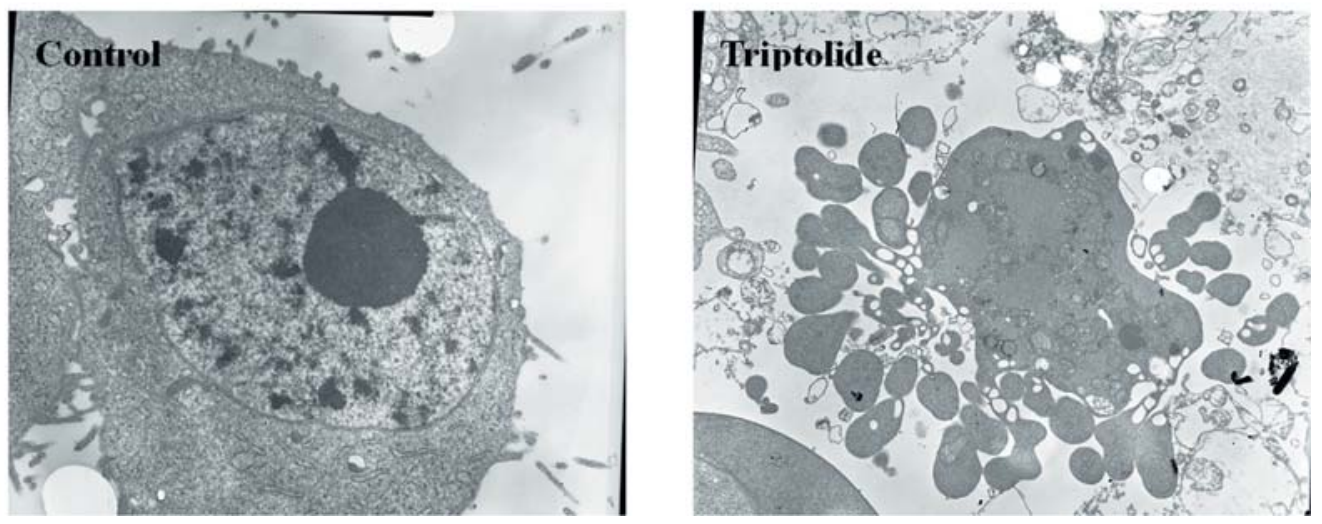

Figure 2. Hoechest 33258 staining (A) and transmission electron microscopy (B) of untreated and triptolide-treated TA-K cells. Cells were treated with 30 nM triptolide for $48 \mathrm{~h}$.

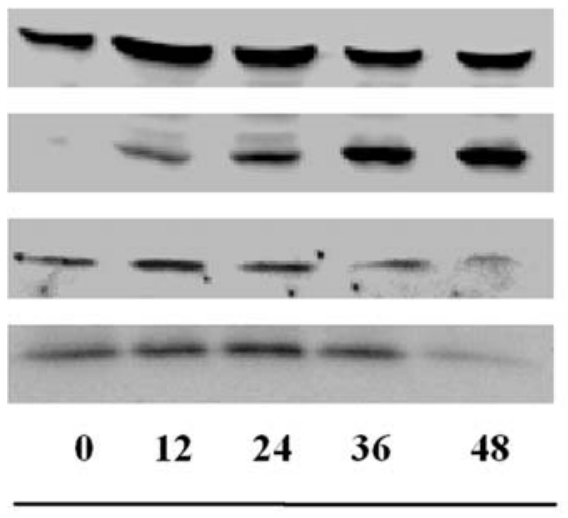

\section{$\beta$-actin}

P53

P21

Bax

(h)

\section{Triptolide (30 nM)}

Figure 3. Effects of triptolide on the protein levels of p53, p21 and Bax in TA-K cells. Cells were treated with $30 \mathrm{nM}$ triptolide for 12, 24, 36 and $48 \mathrm{~h}$ and then harvested for Western blot analysis.

Triptolide increases p53 protein level, but not the protein level of p53 target genes $p 21$ and Bax. In response to the cellular stress such as DNA-damaging agents or radiation, p53 promotes apoptosis via regulating the transcription of cell cycle and apoptosis-related genes (9). To clarify whether triptolide-induced apoptosis is mediated by p53 signaling pathway, the protein level of p53 and its regulated effectors such as p21 and Bax were examined. Fig. 3 shows that triptolide triggered a time-dependent increasing level of p53 protein, but no such tendency occurred in p21 and Bax protein levels. Instead, p21 and Bax proteins even slightly reduced at $48 \mathrm{~h}$, accompanied with an obvious enhanced p53 protein expression. These data indicate that the p53 pathway was not successfully activated.

Triptolide decreases the expression of 065 (the active subunit of $N F-\kappa B$ ) and $N F-\kappa B$ downstream genes Bcl-2 and $-X_{L}$. Considering the predominant role of $N F-\kappa B$ pathway in cancer cell survival, we probed the alteration of NF- $\mathrm{KB}$ and its targeting apoptosis-associated genes in response to triptolide. Western blot analysis identified that the protein level of p65, the active subunit of $\mathrm{NF}_{-} \mathrm{\kappa B}$, was stable until $36 \mathrm{~h}$ and then markedly declined (Fig. 4A). The protein levels of Bcl-2 and $\mathrm{Bcl}-\mathrm{X}_{\mathrm{L}}$ also began to decrease from $36 \mathrm{~h}$, showing the same trend as p65 (Fig. 4A). Meanwhile, this effect of triptolide on $\mathrm{p} 65, \mathrm{Bcl}-2$ and $\mathrm{Bcl}-\mathrm{X}_{\mathrm{L}}$ protein expressions appears to be dose-dependent and was maximal at concentration of $30 \mathrm{nM}$ (Fig. 4B). Our results support the notion that the NF-кB signal is definitely down-regulated in the process of cell apoptosis induced by triptolide. 
A

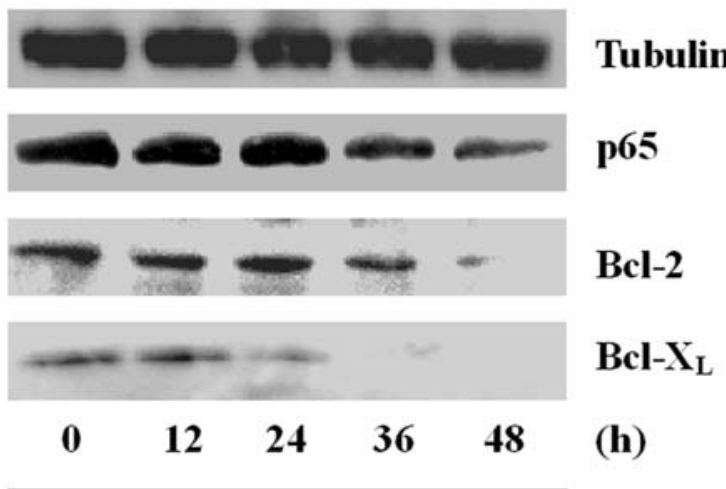

Triptolide (30 nM)

\section{B}

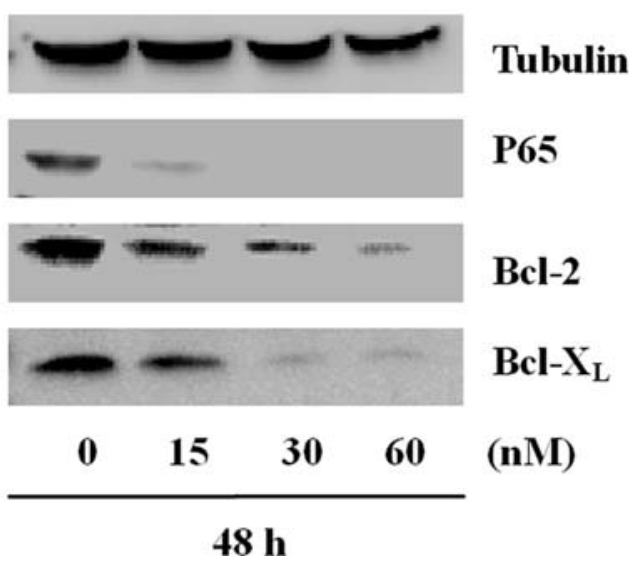

Figure 4. Effects of triptolide on the protein levels of p65, Bcl-2 and Bcl- $\mathrm{X}_{\mathrm{L}}$. (A) The time-dependent effect of triptolide on the protein level of p65, Bcl-2 and Bcl- $\mathrm{X}_{\mathrm{L}}$. Cells were treated with $30 \mathrm{nM}$ triptolide for 12, 24, 36 and $48 \mathrm{~h}$. (B) The concentration-dependent effect of triptolide on the protein levels of p65, Bcl-2 and Bcl- $X_{\mathrm{L}}$. Cells were treated with $0,15,30$ and $60 \mathrm{nM}$ triptolide for $48 \mathrm{~h}$.

\section{Discussion}

ATC is a highly aggressive malignant cancer and has a dismal prognosis because of its rapid growth and early invasion. Strategies for attacking uncontrolled tumor cells to induce apoptosis using small molecular drugs have emerged from an understanding of how tumor cells escape cell death. In our study, we show that triptolide, a natural small molecular product, significantly induces apoptosis of human anaplastic thyroid carcinoma cells. It is well known that p53 and $\mathrm{NF}-\kappa \mathrm{B}$ are common transcriptional factors which have the ability to modulate the expression of apoptosis relevant proteins (18). To clarify the mechanism by which triptolide induces cell apoptosis, we therefore probed its effects on two pathways and found that triptolide does not activate the p53 pathway, but indeed inhibits the $\mathrm{NF}-\kappa \mathrm{B}$ pathway. Taken together, triptolide promotes apoptosis of TA-K cells by down-regulating NF- $\mathrm{KB}$ and the targeting gene expression.
Wild-type p53 is functional to trigger cell apoptosis in the circumstance of cell stress (9). However, p53 gene is inactivated/unavailable in approximately half of tumors and p53 mutation/depletion results in the failure of most cancers to respond to radiotherapy and chemotherapy $(19,20)$. There is evidence to support the notion that restoration of p53 function leads to the commitment of tumor cells to apoptosis (21). In this study, it is of interest that up-regulation of p53 downstream genes such as p21 and Bax is not seen in conjunction with up-regulation of p53 by triptolide in our anaplastic thyroid cells. This is in contrast to the upregulation of these genes that generally accompanies p53mediated apoptosis in response to DNA damaging drugs, but consistent with previous studies on triptolide-induced apoptosis (22). Increasing p53 protein accompanied with decreased cell cycle arrest/apoptosis regulators p21 and Bax expression suggest that apoptosis triggered by triptolide is not mediated by the p53 pathway. Additionally, it is tempting to speculate that triptolide recovers the protein level of p53, but not the transcriptional function or triptolide regulates p21 and Bax expression via a p53-independent mechanism, thus further investigation is required in additional experimental models.

The overactivation of $\mathrm{NF}-\kappa \mathrm{B}$ plays a major role in tumor pathogenesis, allowing neoplastic cells to survive and providing a promising target for the development of chemotherapy agents (11). Bcl-2 and $\mathrm{Bcl}-\mathrm{X}_{\mathrm{L}}$ are $\mathrm{NF}-\kappa \mathrm{B}$-induced members of the Bcl-2 family and exert their protecting effects on apoptosis through stabilization of the mitochondrion $(6,23)$. In addition, basal level of $\mathrm{Bcl}-\mathrm{X}_{\mathrm{L}}$ but not $\mathrm{Bax}$ and $\mathrm{Bcl}-2$ shows a strong negative correlation with the sensitivity to cytotoxic agents (24), indicating $\mathrm{Bcl}-\mathrm{X}_{\mathrm{L}}{ }^{\prime} \mathrm{s}$ unique role in determining the survival of cancer cells exposed to chemotherapeutic agents. Accumulating research demonstrates that targeting $\mathrm{NF}-\kappa \mathrm{B}$ and its downstream genes Bcl-2 and $\mathrm{Bcl}-\mathrm{X}_{\mathrm{L}}$ is effective in causing cytotoxicity and thus achieving the goal of tumor cell killing $(25,26)$. Our results show that triptolide leads to reduction of $\mathrm{NF}-\kappa \mathrm{B}$ active unit $\mathrm{p} 65$ and anti-apoptotic protein $\mathrm{Bcl}-2$ and $\mathrm{Bcl}-\mathrm{X}_{\mathrm{L}}$ and the extent of reduction is stronger than Bax. These data reveal that the increased ratio of Bax:Bcl-2/- $\mathrm{X}_{\mathrm{L}}$ may have a role in the induction of apoptosis by triptolide, and $\mathrm{Bcl}-2$ and $\mathrm{Bcl}-\mathrm{X}_{\mathrm{L}}$ exert the principal efficiency.

In summary, our data reveal that triptolide induces human ATC cell apoptosis through downregulating p65 expression and anti-apoptotic proteins Bcl-2 and Bcl- $\mathrm{X}_{\mathrm{L}}$ which are transcriptionally regulated by NF- $\mathrm{B}$, not through p53 signaling pathway. Considering the high mutation rate of $\mathrm{p} 53$, triptolideinduced apoptosis in a p53-independent pathway is beneficial to evade drug resistance, thus providing a promising new therapeutic agent for the treatment of ATC and even other tumors.

\section{Acknowledgements}

This study was supported by Key program, National Natural Science Foundation of China (No. 30830111), National Natural Science Foundation of China (No. 330801408) and National Natural Science Foundation of Guangdong Province (No. 8451008901000297). 


\section{References}

1. Neff RL, Farrar WB, Kloos RT and Burman KD: Anaplastic thyroid cancer. Endocrinol Metab Clin North Am 37: 525-538, 2008.

2. Pasieka JL: Anaplastic thyroid cancer. Curr Opin Oncol 15: 78-83, 2003.

3. McIver B, Hay ID, Giuffrida DF, et al: Anaplastic thyroid carcinoma: a 50-year experience at a single institution. Surgery 130: 1028-1034, 2001.

4. Ain KB: Anaplastic thyroid carcinoma: behavior, biology, and therapeutic approaches. Thyroid 8: 715-726, 1998.

5. Evan GI and Vousden KH: Proliferation, cell cycle and apoptosis in cancer. Nature 411: 342-348, 2001.

6. Burlacu A: Regulation of apoptosis by Bcl-2 family proteins. J Cell Mol Med 7: 249-257, 2003.

7. Hockenbery DM: bcl-2 in cancer, development and apoptosis. J Cell Sci (Suppl) 18: 51-55, 1994.

8. Bykov VJ and Wiman KG: Novel cancer therapy by reactivation of the p53 apoptosis pathway. Ann Med 35: 458-465, 2003.

9. Amundson SA, Myers TG and Fornace AJ Jr: Roles for p53 in growth arrest and apoptosis: putting on the brakes after genotoxic stress. Oncogene 17: 3287-3299, 1998.

10. Aggarwal BB: Nuclear factor-kappaB: the enemy within. Cancer Cell 6: 203-208, 2004

11. Karin M: Nuclear factor-kappaB in cancer development and progression. Nature 441: 431-436, 2006.

12. Tao $X$ and Lipsky PE: The Chinese anti-inflammatory and immunosuppressive herbal remedy Tripterygium wilfordii Hook F. Rheum Dis Clin North Am 26: 29-50, 2000.

13. Shamon LA, Pezzuto JM, Graves JM, et al: Evaluation of the mutagenic, cytotoxic, and antitumor potential of triptolide, a highly oxygenated diterpene isolated from Tripterygium wilfordii. Cancer Lett 112: 113-117, 1997.

14. Tengchaisri T, Chawengkirttikul R, Rachaphaew N, Reutrakul V, Sangsuwan R and Sirisinha S: Antitumor activity of triptolide against cholangiocarcinoma growth in vitro and in hamsters. Cancer Lett 133: 169-175, 1998.

15. Lee KY, Chang W, Qiu D, Kao PN and Rosen GD: PG490 (triptolide) cooperates with tumor necrosis factor-alpha to induce apoptosis in tumor cells. J Biol Chem 274: 13451-13455, 1999.
16. Chan EW, Cheng SC, Sin FW and Xie Y: Triptolide induced cytotoxic effects on human promyelocytic leukemia, T cell lymphoma and human hepatocellular carcinoma cell lines. Toxicol Lett 122: 81-87, 2001

17. Wang CY, Zhong WB, Chang TC, Lai SM and Tsai YF: Tumor necrosis factor alpha induces three-dimensional cytomorphologic differentiation of human anaplastic thyroid carcinoma cells through activation of nuclear factor kappaB. Cancer 95: 1827-1833, 2002.

18. Reed JC: Apoptosis-targeted therapies for cancer. Cancer Cell 3: $17-22,2003$

19. Kastan MB, Canman CE and Leonard CJ: P53, cell cycle control and apoptosis: implications for cancer. Cancer Metastasis Rev 14: 3-15, 1995.

20. Lee JM and Bernstein A: Apoptosis, cancer and the p53 tumour suppressor gene. Cancer Metastasis Rev 14: 149-161, 1995.

21. Yu Q: Restoring p53-mediated apoptosis in cancer cells: new opportunities for cancer therapy. Drug Resist Update 9: 19-25, 2006.

22. Chang WT, Kang JJ, Lee KY, et al: Triptolide and chemotherapy cooperate in tumor cell apoptosis. A role for the p53 pathway. $\mathrm{J}$ Biol Chem 276: 2221-2227, 2001.

23. Kuwana T and Newmeyer DD: Bcl-2-family proteins and the role of mitochondria in apoptosis. Curr Opin Cell Biol 15: 691-699, 2003.

24. Amundson SA, Myers TG, Scudiero D, Kitada S, Reed JC and Fornace AJ Jr: An informatics approach identifying markers of chemosensitivity in human cancer cell lines. Cancer Res 60: 6101-6110, 2000.

25. Ahmad A, Banerjee S, Wang Z, Kong D and Sarkar FH: Plumbagin-induced apoptosis of human breast cancer cells is mediated by inactivation of NF-kappaB and Bcl-2. J Cell Biochem 105: 1461-1471, 2008 .

26. Pozo-Guisado E, Merino JM, Mulero-Navarro S, et al: Resveratrol-induced apoptosis in MCF-7 human breast cancer cells involves a caspase-independent mechanism with downregulation of Bcl-2 and NF-kappaB. Int J Cancer 115: 74-84, 2005. 